\title{
RESEARCH
}

Open Access

\section{Correlation between frataxin expression and contractility revealed by in vitro Friedreich's ataxia cardiac tissue models engineered from human pluripotent stem cells}

Andy On-Tik Wong ${ }^{1 \dagger}$, Gabriel Wong ${ }^{1+}$, Michael Shen ${ }^{1}$, Maggie Zi-Ying Chow ${ }^{1}$, Wan Wai Tse ${ }^{1}$, Bimal Gurung ${ }^{1}$, Suet Yee Mak', Deborah K. Lieu' ${ }^{1}$, Kevin D. Costa', Camie W. Chan ${ }^{1}$, Alain Martelli', Joseph F. Nabhan ${ }^{2 *}$ and Ronald A. $\mathrm{Li}^{1^{*}}$

\begin{abstract}
Background: Friedreich's ataxia (FRDA) is an autosomal recessive disease caused by a non-coding mutation in the first intron of the frataxin (FXN) gene that suppresses its expression. Compensatory hypertrophic cardiomyopathy, dilated cardiomyopathy, and conduction system abnormalities in FRDA lead to cardiomyocyte (CM) death and fibrosis, consequently resulting in heart failure and arrhythmias. Murine models have been developed to study disease pathology in the past two decades; however, differences between human and mouse physiology and metabolism have limited the relevance of animal studies in cardiac disease conditions. To bridge this gap, we aimed to generate species-specific, functional in vitro experimental models of FRDA using 2-dimensional (2D) and 3-dimensional (3D) engineered cardiac tissues from FXN-deficient human pluripotent stem cell-derived ventricular cardiomyocytes (hPSC-hvCMs) and to compare their contractile and electrophysiological properties with healthy tissue constructs.
\end{abstract}

Methods: Healthy control and FRDA patient-specific hPSC-hvCMs were derived by directed differentiation using a small molecule-based protocol reported previously. We engineered the hvCMs into our established human ventricular cardiac tissue strip (hvCTS) and human ventricular cardiac anisotropic sheet (hvCAS) models, and functional assays were performed on days 7-17 post-tissue fabrication to assess the electrophysiology and contractility of FRDA patient-derived and FXN-knockdown engineered tissues, in comparison with healthy controls. To further validate the disease model, forced expression of FXN was induced in FXN-deficient tissues to test if disease phenotypes could be rescued.

(Continued on next page)

\footnotetext{
* Correspondence: jf.nabhan@gmail.com; ronald.li@novoheart.com

${ }^{\dagger}$ Andy On-Tik Wong and Gabriel Wong are joint first authors

${ }^{2}$ Rare Disease Research Unit, Worldwide Research and Development, Pfizer,

610 Main Street, Cambridge, MA 02139, USA

${ }^{1}$ Novoheart, Vancouver, British Columbia V6C 2V6, Canada
}

(c) The Author(s). 2019 Open Access This article is distributed under the terms of the Creative Commons Attribution 4.0 International License (http://creativecommons.org/licenses/by/4.0/), which permits unrestricted use, distribution, and reproduction in any medium, provided you give appropriate credit to the original author(s) and the source, provide a link to the Creative Commons license, and indicate if changes were made. The Creative Commons Public Domain Dedication waiver (http://creativecommons.org/publicdomain/zero/1.0/) applies to the data made available in this article, unless otherwise stated. 
(Continued from previous page)

Results: Here, we report for the first time the generation of human engineered tissue models of FRDA cardiomyopathy from hPSCs: FXN-deficient hvCTS displayed attenuated developed forces (by 70-80\%) compared to healthy controls. High-resolution optical mapping of hvCAS with reduced FXN expression also revealed electrophysiological defects consistent with clinical observations, including action potential duration prolongation and maximum capture frequency reduction. Interestingly, a clear positive correlation between FXN expression and contractility was observed $(\rho>0.9)$, and restoration of FXN protein levels by lentiviral transduction rescued contractility defects in FXN-deficient hvCTS.

Conclusions: We conclude that human-based in vitro cardiac tissue models of FRDA provide a translational, disease-relevant biomimetic platform for the evaluation of novel therapeutics and to provide insight into FRDA disease progression.

\section{Background}

Friedreich's ataxia (FRDA) is a hereditary neuromuscular degenerative disease caused by a trinucleotide repeat expansion mutation in the first intron of the frataxin (FXN) gene [1]. Although FXN, a mitochondrial protein involved in the biosynthesis of iron-sulfur co-factors required by multiple mitochondrial and extra-mitochondrial proteins, is fully functional in FRDA patients, the intronic mutation significantly reduces the expression to only 5 to $30 \%$ of non-carriers $[2,3]$. Clinically, the heart is a major site of pathology in FRDA: cardiac symptoms are first diagnosed as an abnormal electrocardiogram (EKG) and can progress to compensatory hypertrophic cardiomyopathy and dilated cardiomyopathy, followed by cardiomyocyte (CM) death and fibrosis, ultimately leading to arrhythmias and heart failure [4-7]. For FRDA patients, cardiac dysfunction is the leading cause of death [8].

With advances in reprogramming, human induced pluripotent stem cells (hiPSCs) have been derived from FRDA patients and differentiated into CMs to study the disease progression in a human in vitro model. Previous single-cell studies using FRDA hiPSC-derived CMs have demonstrated cellular phenotypes such as hypertrophy, accumulation of intracellular iron and reactive oxygen species (ROS), attenuated ATP production, and compromised $\mathrm{Ca}^{2+}$ handling consistent with patient symptoms [9-12]. However, considering that the heart is a 3-dimensional (3D) dynamic organ whose primary function is to pump, no multicellular model has been reported to study FRDA disease phenotypes at the tissue level, such as the impact on contractility. It is generally acknowledged that observations from 2dimensional (2D) biological assays do not necessarily translate to $3 \mathrm{D}$ systems $[13,14]$, and $3 \mathrm{D}$ cardiac engineered tissues have proven valuable in modeling cardiac diseases including hypertrophic cardiomyopathy $[15,16]$. Here, we investigated for the first time the effects of reduced FXN level on cardiac electrophysiological and contractile properties with various engineered tissue constructs fabricated from normal and FXN-deficient human embryonic stem cell (hESC)- and induced pluripotent stem cell (hiPSC)- derived ventricular cardiomyocytes (hvCMs). Specifically, our advanced engineered tissue platform permits generation of human ventricular cardiac anisotropic sheets (hvCAS), monolayers of strategically aligned hvCMs to reproduce structural and functional anisotropy of the native ventricle [17-19]. Using hvCAS, we measured cellular action potential parameters and electrical conduction as a syncytium, and with 3D human ventricular cardiac tissue strips (hvCTS) that resemble cardiac trabecular muscles $[16,20,21]$, we evaluated contractility. Collectively, these hvCAS and hvCTS constructs offer a cardiomimetic in vitro model system which yields multicellular readouts such as conduction velocity and magnitude of force generation that are otherwise not possible in single-cell models. Our results are discussed in relation to their mechanistic and translational insights into FRDA.

\section{Methods}

hPSC culture and differentiation into CMs

FRDA patient-specific hiPSCs (FRDA(68) and FRDA(03665)) were obtained from the University of Alabama at Birmingham. Healthy hESCs (HES2; ESI, NIH code ES02) and FRDA(03665) were cultured on hESCqualified Matrigel (Corning) with mTeSR1 medium (Stem Cell Technologies). Control hiPSCs (PB02), reprogrammed from peripheral blood mononuclear cells by episomal nucleofection of transcription factors-OCT3/ 4, SOX2, KLF4, L-MYC, and LIN28-plus p53interfering shRNA, and $\operatorname{FRDA}(68)$ were cultured on hESC-qualified Geltrex (Gibco) with Essential 8 Medium (Gibco). All hPSC lines were cultured at $37^{\circ} \mathrm{C}$ with $5 \%$ $\mathrm{CO}_{2}$. To differentiate hPSCs into cardiomyocytes, dissociated hPSCs were allowed to form cell clusters in either mTeSR1 or Essential 8 (same as in regular culture) with Matrigel and $1 \mathrm{ng} / \mathrm{ml}$ bone morphogenetic protein 4 (BMP4) overnight in an ultra-low attachment plate and hypoxic condition. From day 1 to 4 , cell clusters were treated with $50 \mu \mathrm{g} / \mathrm{ml}$ ascorbic acid (Sigma-Aldrich), $10 \mathrm{ng} / \mathrm{ml}$ activin A, $10 \mathrm{ng} / \mathrm{ml} \mathrm{BMP4}$, and $10 \mu \mathrm{M}$ ROCK inhibitor Y-27632 in StemPro-34 medium 
supplemented by GlutaMAX (Thermo Fisher Scientific) in hypoxic condition. Next, cell clusters in hypoxic condition were treated with $50 \mu \mathrm{g} / \mathrm{ml}$ ascorbic acid and 5 mM IWR-1 in StemPro-34 medium until day 8. Cell clusters were maintained after day 8 in normoxic condition with StemPro-34 media containing $50 \mu \mathrm{g} / \mathrm{ml}$ ascorbic acid. Using this differentiation protocol [22], ventricular subtype yield of over 70\% of hPSC-derived CMs was achieved. These differentiated cells are referred to as human ventricular (hv) CM and were used in all experiments presented in this study. Differentiation yield from hESC and hiPSC lines in this study had a median of $73-77 \%$, as estimated by the percentage of cardiac troponin T-positive cells (Additional file 1: Figure S1).

\section{Knockdown and overexpression of FXN in hvCMs}

To model FXN deficiency of FRDA in hESC- and hiPSC-derived hvCMs, FXN was knocked down in both types of hvCMs by transduction with lentiviral shRNA (Lv-shFXN1: TRCN0000006137 or LvshFXN2: TRCN0000010996 inserts in pLKO.1 vector backbone) at MOI of 5 following the timeline in Fig. 1a. Respective control hvCMs were transduced with mammalian non-targeting shRNA (Lv-shNT). To restore FXN expression, FXN-deficient hvCMs were transduced with lentivirus to overexpress FXN (LvFXN; GE Dharmacon OHS5835-EG2395), with lentivirus delivering red fluorescent protein (Lv-RFP) serving as a control. To assess and compare the electrophysiological and contractile functions among healthy, FXN-deficient, and/or FXN-overexpressing hvCMs, two tissue construct platforms were constructed from these cells, each specifically designed to enable functional assessment as described below. Control hiPSC groups were not virally transduced except for where specified otherwise.

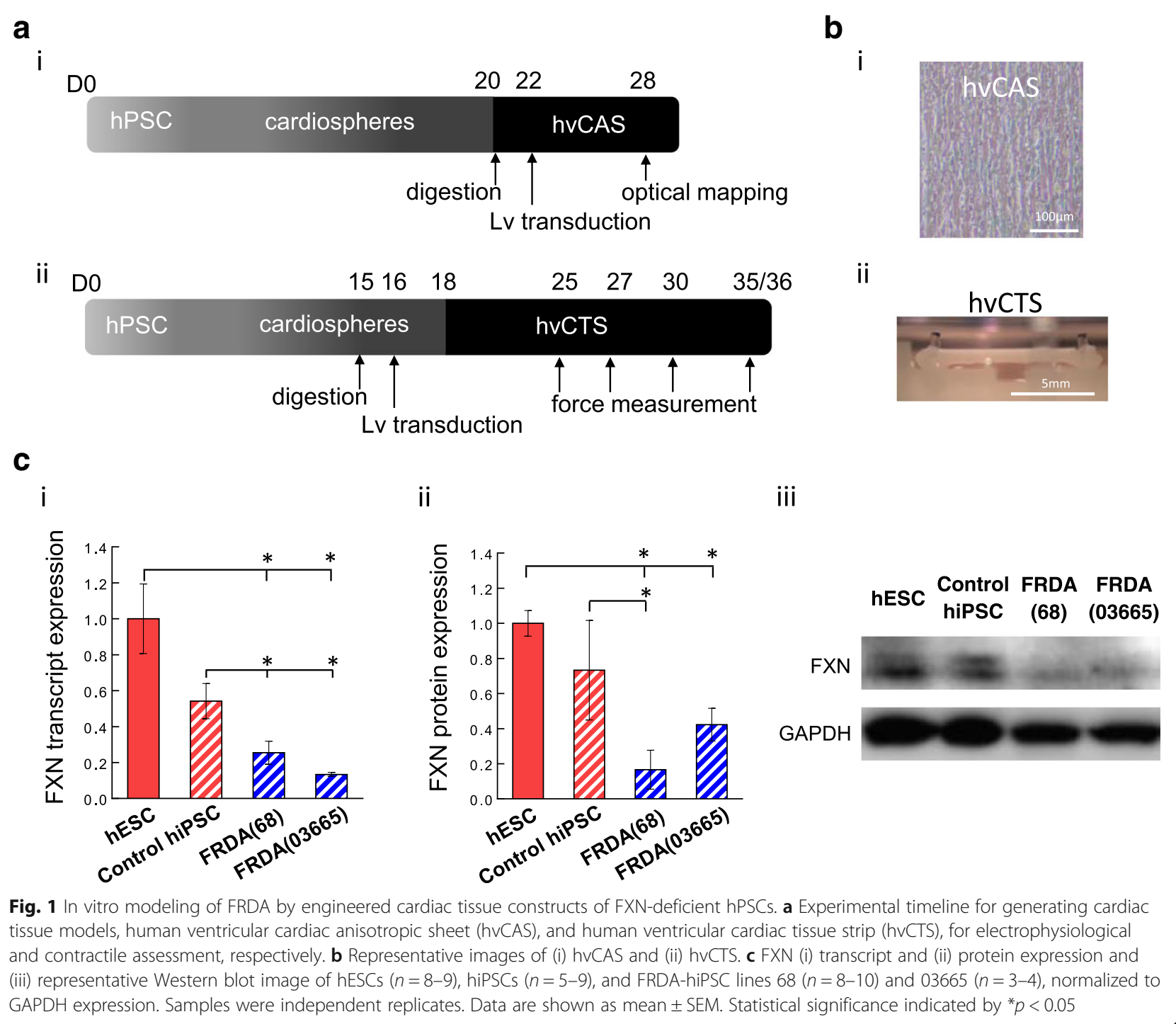




\section{Contractile assessment of human ventricular cardiac tissue strips}

To assess hvCM contractile function by direct measurement of contractile force generation, 3D multicellular hvCTS myocardial tissues were engineered as previously described [20, 21]. Briefly, cardio-clusters from day 15 of hPSC cardiac differentiation were dissociated into single cells and allowed to recover in the incubator for 3 days before hvCTS construction. Each hvCTS consisted of $1.3 \times 10^{6}$ cardiac cells differentiated from hPSCs and $1.3 \times 10^{5}$ human foreskin fibroblasts in a $100-\mu l$ ice-cold solution of $2 \mathrm{mg} / \mathrm{ml}$ collagen I (Thermo Fisher Scientific), 0.80-0.95 mg/ml Matrigel, 0.6× PBS, $20 \mathrm{mM} \mathrm{NaOH}, 0.8 \times$ Minimum Essential Medium (Sigma-Aldrich), $1.6 \mathrm{mM}$ HEPES, and $0.1 \times$ hvCTS maintenance medium (see composition below). A volume of $100 \mu \mathrm{l}$ of the final cellcollagen mixture is then added to each polydimethylsiloxane (PDMS) bioreactor, consisting of a force-sensing cantilever post at each end of a rectangular well, and returned to the incubator to form the hvCTS attached between the two end posts. The hvCTS were maintained in DMEM medium supplemented with 10\% newborn calf serum (Gibco), with daily half-medium changes, until ready for testing.

Force generated by the hvCTS was measured at $37^{\circ} \mathrm{C}$ in phenol red-free DMEM medium with HEPES buffer using either a custom-designed post-tracking force measurement system that records displacement of the cantilever posts on a temperature-controlled heating plate, or in a commercial isometric muscle bath system (Aurora Scientific) at the end of the study, as specified in the "Results" section. During testing, the hvCTS were paced by electrical field stimulation at $1 \mathrm{~Hz}$ frequency. An overview of the experimental timeline is shown in Fig. 1a (ii). For longitudinal studies, the hvCTS were returned to the incubator with fresh media following testing at intermediate timepoints.

\section{Electrophysiological assessment of human ventricular cardiac anisotropic sheets}

To assess the electrophysiological properties of hvCMs as an aligned and electrically coupled syncytium with anisotropic conduction property similar to ventricular $\mathrm{CMs}$ in vivo, cardio-clusters from day 20 of hPSC cardiac differentiation were dissociated into single cells, and $4.5 \times 10^{5}$ cells/ $\mathrm{cm}^{2}$ were plated as a monolayer on Matrigel-coated, microgrooved substrates fabricated from polystyrene shrink film (Shrinky Dinks "Crystal Clear," K\&B Innovations), with groove width of $15 \mu \mathrm{m}$, groove depth of $5 \mu \mathrm{m}$, and intergroove distance of $5 \mu \mathrm{m}$, to form hvCAS as previously described [18]. After allowing recovery for 8 days, action potentials of hvCAS were optically mapped with a MiCAM ULTIMA imaging system (SciMedia) using voltage-sensitive fluorophore Di-8-ANEPPS with Pluronic F-127 (Thermo
Fisher Scientific) in Tyrode's solution containing blebbistatin (Sigma-Aldrich). Automaticity, threshold voltage, and maximum capture frequency (MCF) were first determined for each hvCAS, followed by programmed electrical stimulation to test for reentrant arrhythmias. An overview of the experimental timeline is shown in Fig. 1a (i).

\section{Results \\ FRDA hiPSCs exhibited reduced FXN expression}

To create human engineered cardiac tissue models of FRDA, hPSCs were differentiated into hvCMs and fabricated into hvCAS and hvCTS, following a defined schedule (Fig. 1a, b). FXN expression was first assessed in four hPSC lines, including one healthy hESC, one healthy hiPSC, and two FRDA-hiPSC lines-FRDA(68) and FRDA(03665)-reprogrammed from two FRDA patients. FXN at the transcript level was comparable for the two FRDAhiPSC lines, with both expressing $<50 \%$ of the FXN levels observed in the healthy hESC and hiPSC lines (Fig. 1c (i)). The protein level for FXN was in agreement with the transcripts, with FRDA-hiPSCs expressing the lowest level of FXN relative to the healthy hPSC lines (Fig. 1c (ii, iii)).

\section{Isogenic FXN-knockdown models of hESC-hvCMs exhibited reduced FXN expression and contractile function}

To model FRDA in isogenic human CMs in vitro, hESCderived hvCMs were transduced with the lentiviral construct Lv-shFXN1 or Lv-shFXN2 for shRNA-mediated FXN suppression or with non-targeting Lv-shNT as control. Both Lv-shFXNs successfully induced FXN knockdown at the transcript level, as demonstrated by 50\% reduced transcript expression in hESC-vCMs relative to Lv-shNT-transduced control (Fig. 2a (i)). The reduction in FXN protein in hvCMs was $40 \%$ and $70 \%$ for $\mathrm{Lv}$ shFXN1 and Lv-shFXN2, respectively (Fig. 2a (ii, iii)). Using these transduced hESC-hvCMs to construct hvCTS, contractility was then assessed as developed force generation during electrical field stimulation at $1 \mathrm{~Hz}$ on days 7 , 9, and 12 after tissue fabrication (Fig. 2b, c). Contractile force in control hESC-hvCTS progressively increased from a median of $5 \mu \mathrm{N}$ on day 7 to $135 \mu \mathrm{N}$ on day 12. Conversely, hESC-hvCTS with FXN knockdown by either Lv-shFXN construct failed to demonstrate a significant increase in developed force over time. By day 12, the median force in FXN-deficient hvCTS was $75-80 \%$ lower than that of control (Fig. 2c (iii)). In addition, the contraction and relaxation rates were $>3$ times slower in FXN-deficient hvCTS (Fig. 2d). There was no significant difference in developed force between nontransduced and Lv-shNT hESC-hvCTS (data not shown). 


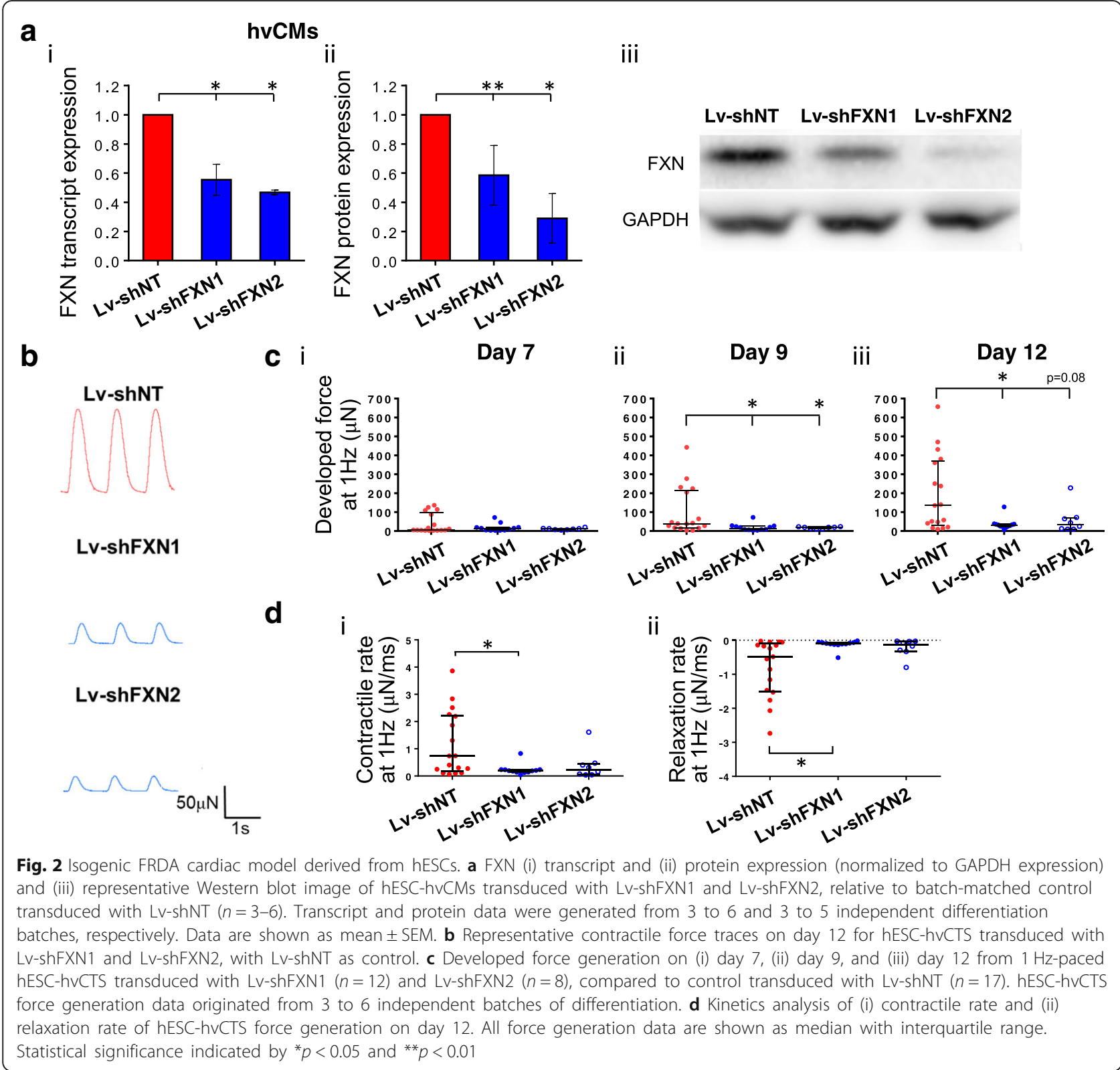

\section{Patient-derived FRDA cardiac models were FXN-deficient} with compromised contractility

FXN expression was assessed at both the transcript and protein levels for hvCMs derived from FRDA(68)hiPSCs, FRDA(03665)-hiPSCs, and healthy hiPSCs. hvCMs differentiated from FRDA(68)-hiPSCs and FRDA(03665)-hiPSCs expressed about 70\% less FXN at the transcript level (Fig. 3a (i)), and nearly 40-60\% less at the protein level, compared to controls from healthy donors (Fig. 3a (ii, iii)). Force was measured after the construction of FRDA-hiPSC-hvCMs into hvCTS. To correlate the force generation to FXN expression, the developed forces in day $12 \mathrm{hvCTS}$ at $1 \mathrm{~Hz}$ of all groups recorded were plotted against the FXN transcript levels (Fig. 3b). Generally, FXN-deficient hvCTS-due to either shRNA-mediated knockdown or intrinsic genetic defect in FRDA-hiPSC-exhibited compromised force generation by developing less than half of the force as healthy controls that expressed robust FXN. As indicated by Pearson's coefficient of 0.96 , there was a strong correlation between the levels of developed force and FXN expression.

\section{Electrophysiological properties were altered in FXN- deficient hvCAS}

Next, the electrophysiological properties of shRNAsuppressed and FRDA hPSC-derived hvCMs were assessed by high-resolution optical mapping of hvCAS 


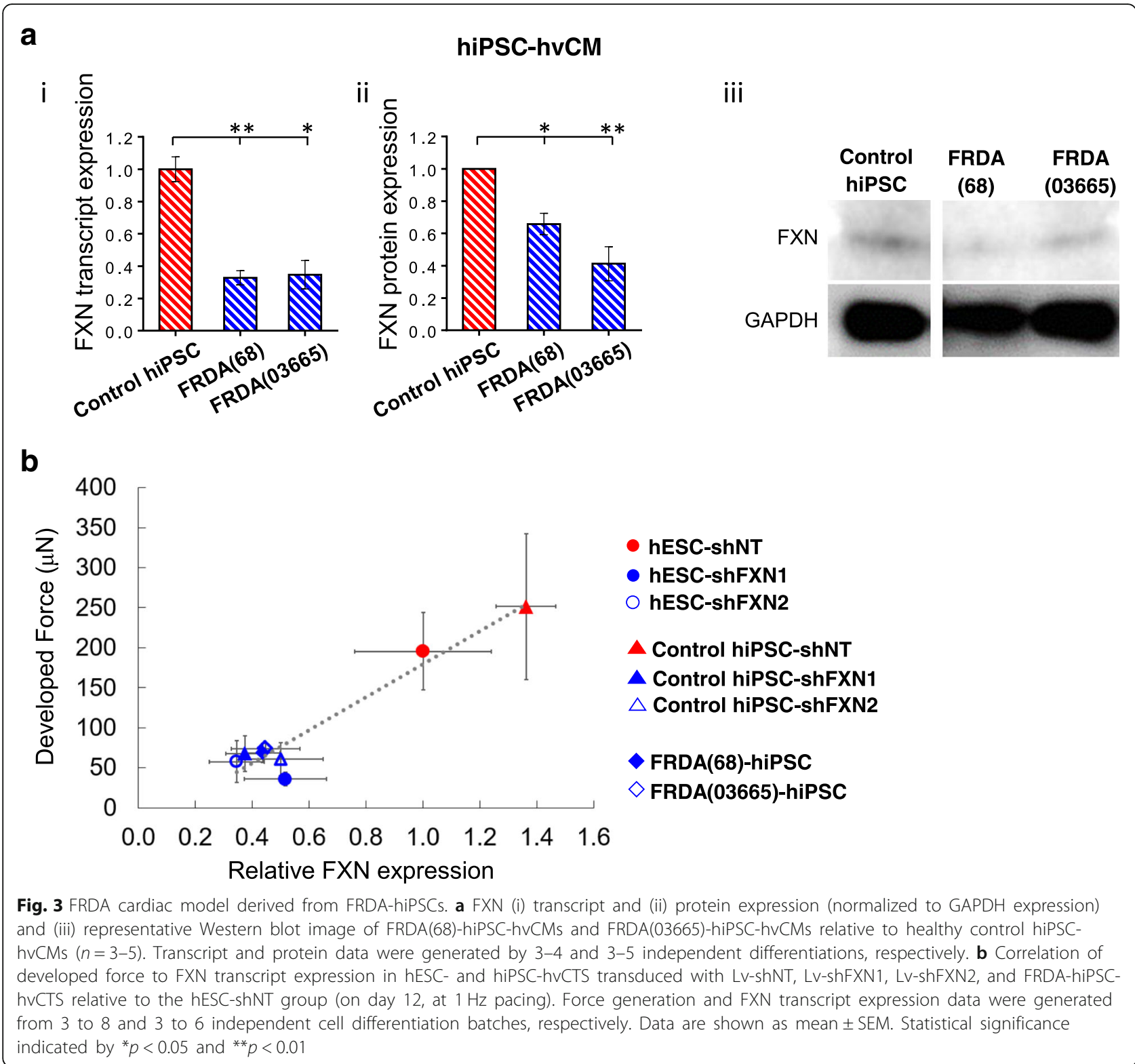

in comparison with the corresponding controls (Fig. 4a). The incidence of spiral conduction waves and automaticity (i.e., spontaneous generation of action potentials) were not statistically different between hESC-hvCAS transduced with Lv-shFXN and LVshNT-transduced control (Additional file 1: Table S1). However, the MCF of Lv-shFXN-transduced hESChvCAS, with a median of $2.0 \mathrm{~Hz}$, was statistically lower than control $(2.5 \mathrm{~Hz}$; Fig. 4b (i)). Similarly, FRDA-hiPSC-derived hvCAS were harder to capture at higher pacing frequency compared to control (median $\mathrm{MCF}=1.5 \mathrm{~Hz}$ for FRDA-hiPSC-hvCAS vs. $2.5 \mathrm{~Hz}$ for healthy hiPSC-hvCAS; Fig. 4b (ii)).
Action potential and conduction parameters revealed further electrophysiological differences between healthy and FXN-deficient hvCAS (Fig. 4c). Action potential upstroke and decay velocities and anisotropic ratio did not show consistent differences between FXN-deficient hvCAS and control for both the hESC and hiPSC groups (Additional file 1: Table S1). However, action potential duration at $50 \%$ repolarization (APD50) and 90\% repolarization (APD90) were significantly prolonged in hESC-hvCAS transduced with Lv-shFXN (APD50, $152 \mathrm{~ms} ;$ APD90, $266 \mathrm{~ms}$ ) relative to control (APD50, $138 \mathrm{~ms}$; APD90, $236 \mathrm{~ms}$ ) (Fig. 4c (i, iii)). Notably, APD50 and APD90 were 


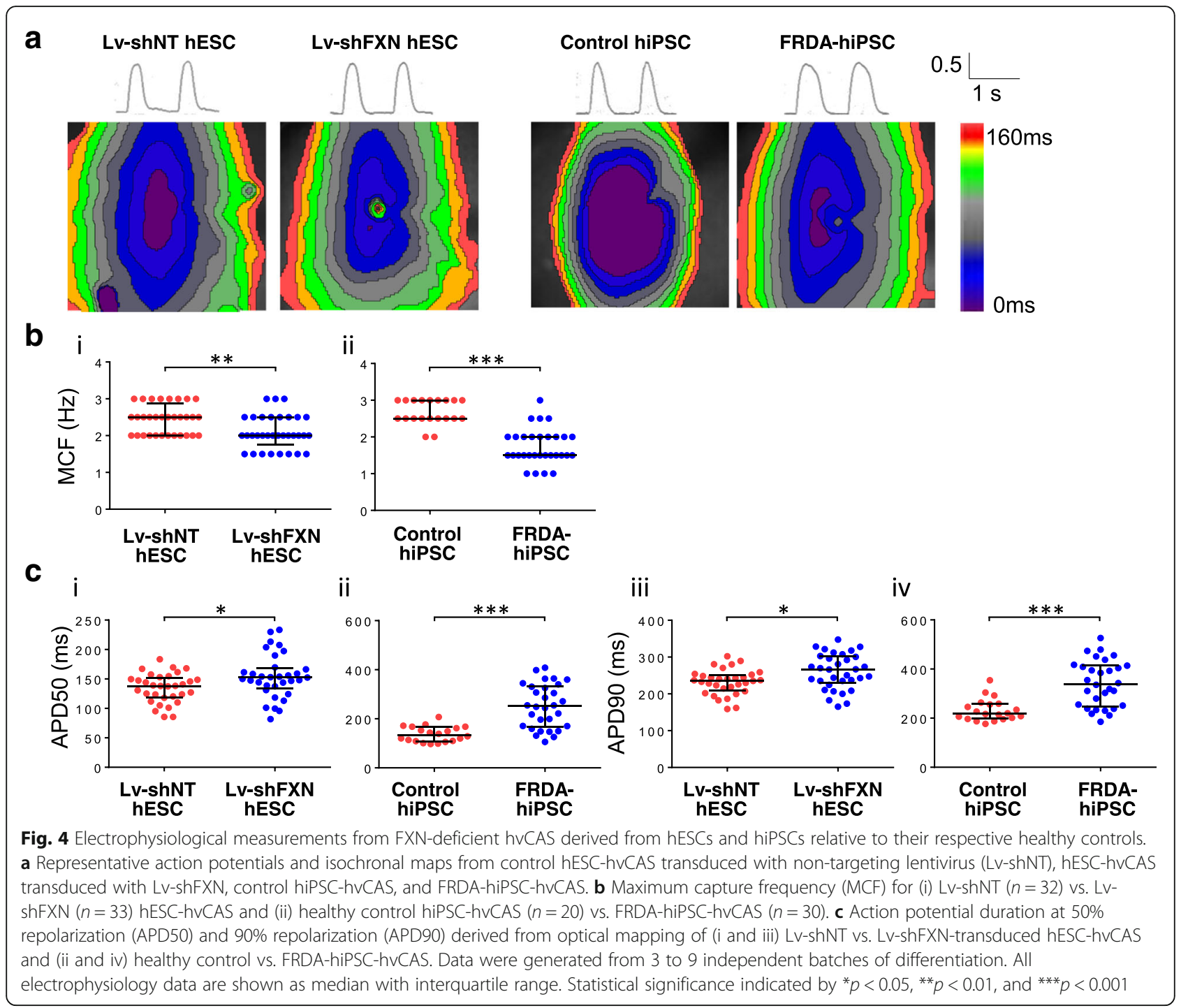

similarly prolonged in FRDA-hiPSC-hvCAS compared to healthy controls (Fig. 4c (ii, iv)).

\section{Restoration of FXN expression rescued hvCTS contractile function}

Considering the strong positive correlation between the developed force and FXN expression, we postulated that restoration of FXN expression would suffice to rescue the compromised contractile function in the FXNdeficient hvCTS. To test this, FXN expression was rescued in FRDA-hvCTS by transduction with Lv-FXN. FXN deficiency in FRDA-hvCTS transduced with LvFXN was rectified as indicated by $\sim 60$-fold and $\sim 40$ fold higher expression of FXN transcript and protein, respectively, compared to control transduced with Lv-RFP (Fig. 5a). Contractile force developed at $1 \mathrm{~Hz}$ pacing in Lv-FXN-transduced FRDA-hvCTS on days 17-18 postconstruction was elevated, with a median of $178 \mu \mathrm{N}$ compared to control of $87 \mu \mathrm{N}$ (Fig. 5b, c (i)). In addition, the contraction and relaxation rates were increased by 3 -fold in the Lv-FXN group compared to Lv-RFP control (Fig. 5c (ii, iii)).

We next tested the functional consequences of restored FXN expression in the complementary isogenic hESChvCTS FRDA model-Lv-shFXN+Lv-FXN group for simultaneous knockdown and forced expression of FXN and Lv-shFXN+Lv-RFP group with FXN knockdown and RFP control. As anticipated, FXN transcript and protein expression were restored for hESC-hvCTS transduced with $\mathrm{Lv}$-shFXN+Lv-FXN, at levels significantly higher than in Lv-shFXN+Lv-RFP-transduced control (Fig. 6a). Further isometric force measurements, using a physiologic muscle bath to stretch the hvCTS length in $0.225 \mathrm{~mm}(2.5 \%$ strain) increments on day 18 post-construction, revealed that the developed force, under $1 \mathrm{~Hz}$ pacing, increased with increasing muscle strip length in both hvCTS groups, 
a

i

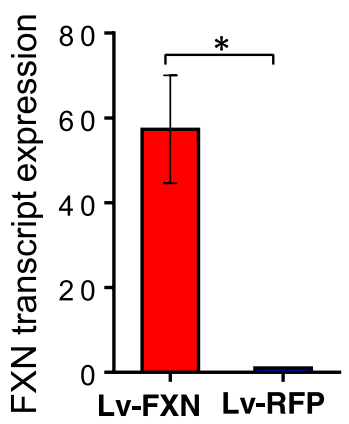

C

i

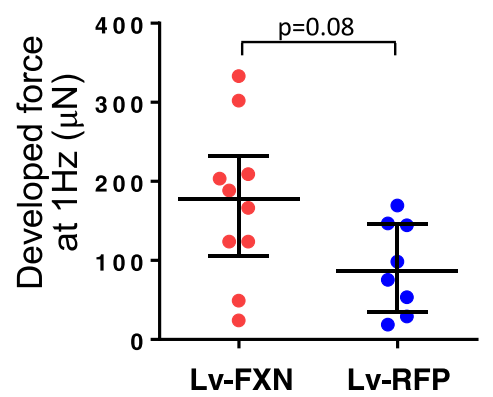

FRDA-hiPSC-hvCTS

ii

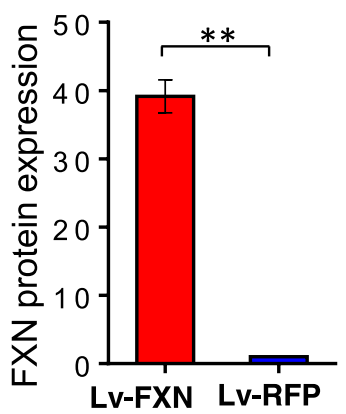

iii

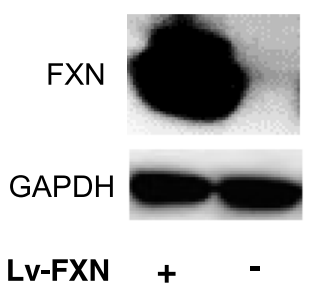

b

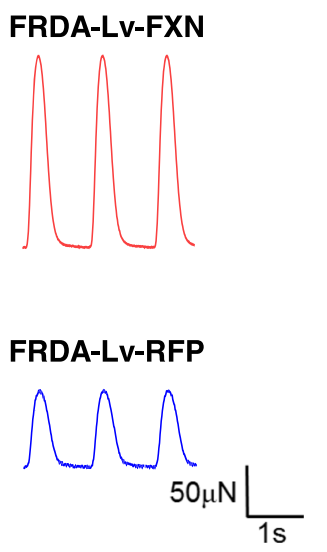

FRDA-hiPSC-hvCTS

ii

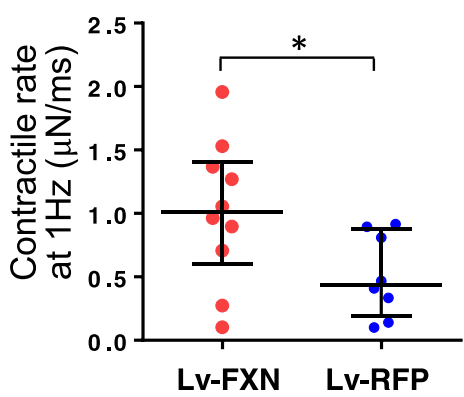

iii

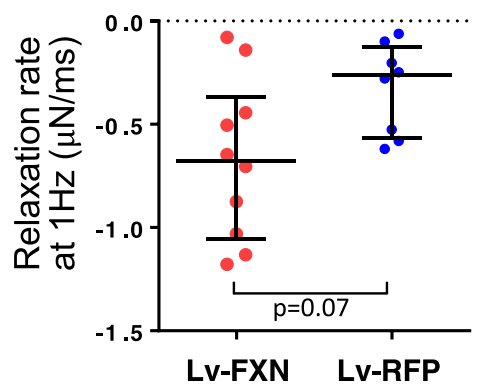

Fig. 5 FXN expression rescue of FRDA hiPSC-hvCTS model. a FXN (i) transcript and (ii) protein expression (normalized to GAPDH expression) and (iii) representative Western blot image of FRDA-hiPSC-hvCTS after Lv-FXN transduction relative to Lv-RFP control $(n=3-4)$. Transcript and protein data were generated from 3 to 4 independent differentiation batches, respectively. $\mathbf{b}$ Representative contractile force traces on days 17-18 for FRDA-hiPSC-hvCTS transduced with Lv-FXN with Lv-RFP as control. c (i) Developed force, (ii) contractile rate, and (iii) relaxation rate generated at $1 \mathrm{~Hz}$ pacing in FRDA-hiPSC-hvCTS transduced with Lv-FXN $(n=10)$ relative to LV-RFP control $(n=8)$. Data were generated by 4 independent batches of cell differentiation. FXN expression data are shown as mean \pm SEM, and force generation data are shown as median with interquartile range. Statistical significance indicated by ${ }^{*} p<0.05$ and ${ }^{* *} p<0.01$

reaching the maximum contractile force at a length defined as $L_{\max }$ (Fig. 6b). Similar to the FXN restorationinduced effects observed in the FRDA-hiPSC model, the isometric developed force was significantly higher in the Lv-shFXN+Lv-FXN hESC-hvCTS FRDA model at strains greater than $90 \% L_{\max }$ (physiological range of $\sim 17.5-$ $27.5 \%$ strain) compared to the control group (Fig. $6 \mathrm{c}$ (i)). At $L_{\text {max }}$, hESC-hvCTS rescued by FXN expression exhibited a mean force of $135 \mu \mathrm{N}$ compared to the FXN-deficient control at $79 \mu \mathrm{N}$. The force development kinetics were also significantly faster in the hESC-hvCTS FRDA model with restored FXN expression, showing a mean contraction rate of $1.04 \mu \mathrm{N} / \mathrm{ms}$ vs. control of $0.64 \mu \mathrm{N} / \mathrm{ms}$ at $L_{\max }$ and a mean relaxation rate of $-0.61 \mu \mathrm{N} / \mathrm{ms}$ vs. control of $-0.35 \mu \mathrm{N} /$ ms at $L_{\max }$ (Fig. 6c (ii, iii)).

\section{Discussion}

Heart failure and arrhythmia are the major causes of mortality in FRDA patients [4-7]. Given the high level of amino acid identity among the FXN genes across species, murine models have been generated for studying FRDA. One murine model based on conditional ablation of mouse Fxn results in complete knockout of $F x n$ in cardiac and skeletal muscle [23]; although this model presents an aggressive FRDA-like phenotype, including cardiomyopathy and mitochondrial defects, it does not mirror the human genotype of trinucleotide repeats, which partially silence expression of the otherwise fully functional protein. Several other mouse models have been generated through transgenic expression of human FXN with expanded GAA repeats, knock-in of a GAA repeat expansion into the mouse $F x n$ locus, or by 


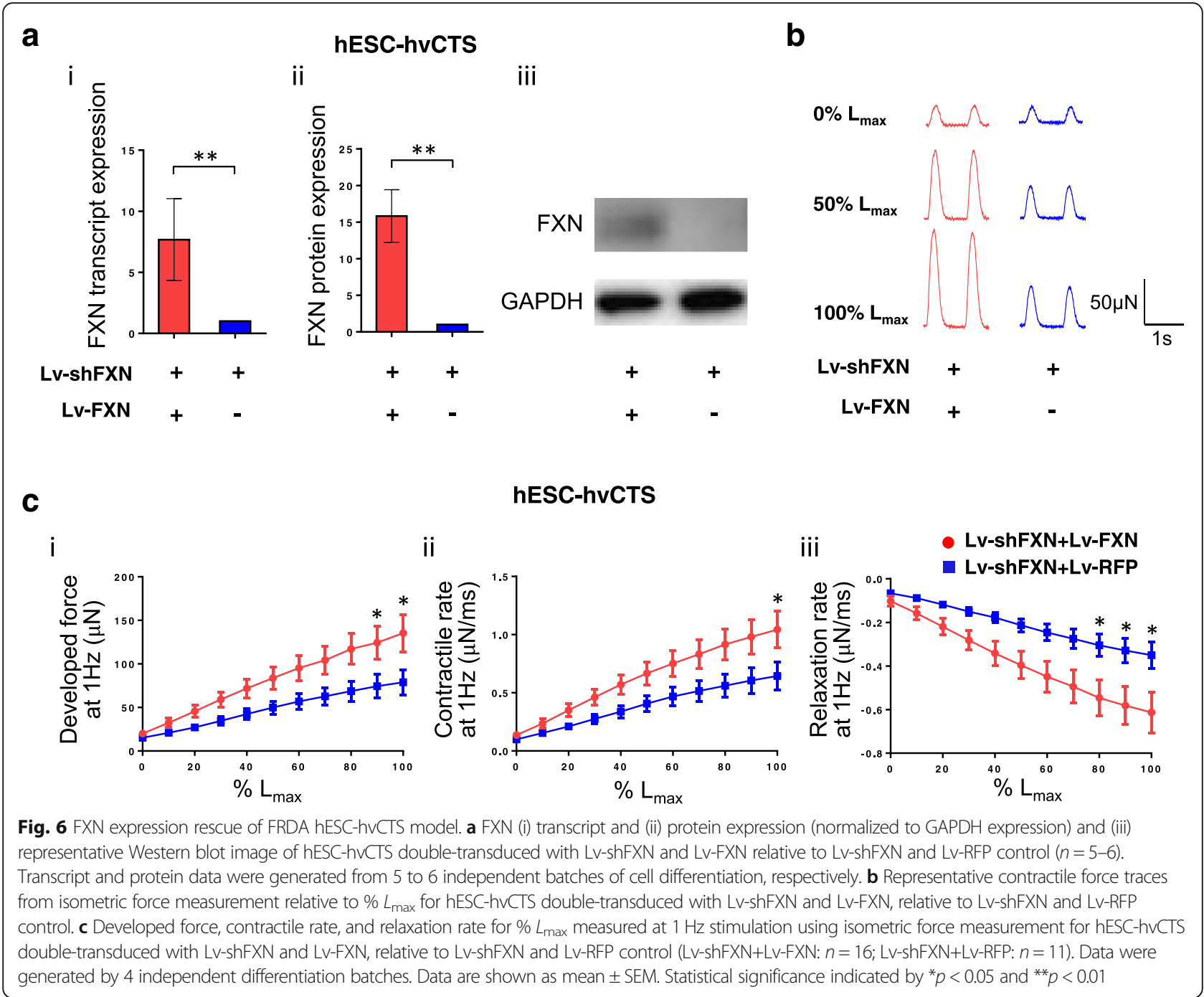

shRNA knockdown of mouse FXN expression, with variable severities of the resultant phenotype [24-26]. Since the use of human models may be more clinically relevant, in the present study we generated engineered human cardiac models of FRDA hvCTS and hvCAS to enable robust in vitro phenotypic readouts.

Despite different genetic backgrounds, similar contractile (weakened developed force) and electrophysiological (reduced MCF and APD prolongation) phenotypes were observed in engineered constructs made out of isogenic hESCs by FXN knockdown or reprogrammed FRDAspecific hiPSCs in which FXN expression is suppressed by pathological GAA expansions. The results suggested a general important role of FXN in cardiobiology. Indeed, a strong positive correlation between developed force and FXN expression was revealed by the collective data of various normal and FRDA hESC/iPSC-hvCTS models. Considering that cardiomyocytes contain the highest mitochondrial density to sustain energy requirement [27] and that FXN promotes the biosynthesis of iron-sulfur clusters, which are co-factors required by mitochondrial proteins such as aconitase and succinate dehydrogenase, reduced levels of FXN are likely to yield adverse effects on mitochondrial function and thereby compromise contractility. Consistently, the attenuated developed force in FRDA hvCTS could be rescued via FXN overexpression. This is in accordance with the inducible and reversible murine FRDA model that demonstrated FXN expression can reverse pathological effects [26]. The exact mechanism that is responsible for the observed disease phenotype and rescue of pathological effects by FXN expression in our human cardiac tissue constructs will need further investigation and may provide novel insights for therapeutic strategies.

Moreover, our work is the only study to date that demonstrated electrophysiological and contractile dysfunction 
from functional assays comparing healthy and FRDA (FXN-deficient) hPSC models. Using bright-field timelapse imaging, beating rate and incidence of arrhythmic activity of control and patient hiPSC-derived cardiomyocytes were previously studied; however, functional data were not reported [9]. Although two publications by Lee et al. $[10,11]$ examined $\mathrm{Ca}^{2+}$-handling properties, no significant differences in $\mathrm{Ca}^{2+}$ transient amplitude and rate of rise or decay were observed between the healthy and FRDA-hiPSC-derived cardiomyocytes. To investigate the efficacy of drugs in clinical trials for treating FRDA, Lee et al. induced pathological effects with the addition of $200 \mu \mathrm{M} \mathrm{Fe}{ }^{2+}$ to both the FRDA and healthy hiPSCderived cardiomyocyte culture. A difference in $\mathrm{Ca}^{2+}$ amplitude and rate of decay between the healthy and FRDA groups was only revealed under such intervention. However, physiological relevance is a concern since healthy cardiomyocytes would not progress to such an ironoverloaded state. Likewise, the results of drug treatments that chelate iron, when tested on iron-overloaded healthy and FRDA hiPSC-cardiomyocytes, may not be predictive of drug efficacy for clinical treatment of FRDA. Therefore, the engineered tissues used in this report without exogenous factors to induce pathological conditions may offer advantages as physiologically relevant models for investigating pathological effects stemming from FXN deficiency and for therapeutic testing.

Electrophysiologically, FXN-deficient hvCAS exhibited action potential prolongation or repolarization delay. The median APD90 prolongation due to FXN deficiency was 30 and $110 \mathrm{~ms}$ in isogenic hESC- and hiPSC-hvCAS models, respectively. These effects are physiologically relevant, as a prolongation of $20 \mathrm{~ms}$ or more in the QT interval is considered to be a high risk for torsade de pointes under FDA guidelines. This observation was also in agreement with Twave inversion or abnormal repolarization of the ventricle recorded in the EKG of FRDA patients that precede the presentation of FRDA cardiomyopathy [7]. Hence, our in vitro human FRDA cardiac tissue model was able to replicate early pathology associated with disease-related cardiomyopathy.

\section{Conclusions}

In summary, we demonstrated that the clinical symptoms of contractile and electrophysiological dysfunction in FRDA patients can be recapitulated by human cardiac tissues engineered from FXN-deficient hPSC-derived hvCMs. Translationally, the positive correlation between FXN expression and contractility and the results of our rescue experiments underscore the potential of FXN restoration by small molecules or gene therapy as an effective therapeutic strategy for suppressing or even reversing the cardiac symptoms of FRDA.

\section{Additional file}

Additional file 1: Figure S1. Percentage yield of cardiomyocytes differentiated from various hESC and hiPSC cell lines. Table S1. Electrophysiological parameters of hvCAS. (PDF $145 \mathrm{~kb}$ )

\section{Abbreviations}

FRDA: Friedreich's ataxia; FXN: Frataxin; hESCs: Human embryonic stem cells; hiPSCs: Human induced pluripotent stem cells; hPSCs: Human pluripotent stem cells; hvCAS: Human ventricular cardiac anisotropic sheets; hvCMs: Human ventricular cardiomyocytes; hvCTS: Human ventricular cardiac tissue strips

\section{Acknowledgements}

We thank Dr. Marek Napierala (University of Alabama at Birmingham) for kindly providing the IPSC lines.

\section{Authors' contributions}

KDC, JFN, and RAL conceived the project. GW, DKL, CWC, KDC, AM, JFN, and RAL supervised the project. AOTW and GW designed the experiments. AOTW, MS, MZYC, WWT, BG, and SYM performed the experiments. All authors contributed to the data analysis and interpretation. AOTW, GW, DKL, KDC, AM, JFN, and RAL contributed to the writing of the manuscript. All authors read and approved the final manuscript.

\section{Funding}

This work was funded by Pfizer and Novoheart.

\section{Availability of data and materials}

All supporting data are included in the article and its additional files.

Ethics approval and consent to participate

Not applicable.

\section{Consent for publication}

Not applicable.

\section{Competing interests}

GW, KDC, CWC, and RAL hold equities in Novoheart whose value may potentially be affected by the publication of this manuscript. AOTW, MS, MZYC, WWT, BG, SYM, and DKL declared that they have no competing interests. AM and JFN were employees of Pfizer Inc. at the time this work was carried out

Received: 7 March 2019 Revised: 30 May 2019

Accepted: 17 June 2019 Published online: 08 July 2019

\section{References}

1. Martelli A, Puccio H. Dysregulation of cellular iron metabolism in Friedreich ataxia: from primary iron-sulfur cluster deficit to mitochondrial iron accumulation. Front Pharmacol. 2014;5:130.

2. Gottesfeld JM. Small molecules affecting transcription in Friedreich ataxia. Pharmacol Ther. 2007;116(2):236-48.

3. Schmucker S, Puccio H. Understanding the molecular mechanisms of Friedreich's ataxia to develop therapeutic approaches. Hum Mol Genet. 2010;19(R1):R103-10

4. Kipps A, Alexander M, Colan SD, Gauvreau K, Smoot L, Crawford L, et al. The longitudinal course of cardiomyopathy in Friedreich's ataxia during childhood. Pediatr Cardiol. 2009;30(3):306-10.

5. Casazza F, Morpurgo M. The varying evolution of Friedreich's ataxia cardiomyopathy. Am J Cardiol. 1996;77(10):895-8.

6. Lynch DR, Regner SR, Schadt KA, Friedman LS, Lin KY, St John Sutton MG. Management and therapy for cardiomyopathy in Friedreich's ataxia. Expert Rev Cardiovasc Ther. 2012;10(6):767-77.

7. Weidemann F, Liu D, Hu K, Florescu C, Niemann M, Herrmann S, et al. The cardiomyopathy in Friedreich's ataxia - new biomarker for staging cardiac involvement. Int J Cardiol. 2015;194:50-7.

8. Tsou AY, Paulsen EK, Lagedrost SJ, Perlman SL, Mathews KD, Wilmot GR, et al. Mortality in Friedreich ataxia. J Neurol Sci. 2011;307(1-2):46-9. 
9. Hick A, Wattenhofer-Donze M, Chintawar S, Tropel P, Simard JP, Vaucamps $\mathrm{N}$, et al. Neurons and cardiomyocytes derived from induced pluripotent stem cells as a model for mitochondrial defects in Friedreich's ataxia. Dis Model Mech. 2013;6(3):608-21.

10. Lee YK, Ho PW, Schick R, Lau YM, Lai WH, Zhou T, et al. Modeling of Friedreich ataxia-related iron overloading cardiomyopathy using patientspecific-induced pluripotent stem cells. Pflugers Arch. 2014;466(9):1831-44.

11. Lee YK, Lau YM, Ng KM, Lai WH, Ho SL, Tse HF, et al. Efficient attenuation of Friedreich's ataxia (FRDA) cardiomyopathy by modulation of iron homeostasis-human induced pluripotent stem cell (hiPSC) as a drug screening platform for FRDA. Int J Cardiol. 2016;203:964-71.

12. Crombie DE, Curl CL, Raaijmakers AJ, Sivakumaran P, Kulkarni T, Wong RC, et al. Friedreich's ataxia induced pluripotent stem cell-derived cardiomyocytes display electrophysiological abnormalities and calcium handling deficiency. Aging (Albany NY). 2017;9(5):1440-52.

13. Passier R, Orlova V, Mummery C. Complex tissue and disease modeling using hiPSCs. Cell Stem Cell. 2016;18(3):309-21.

14. Kawaguchi N, Hatta K, Nakanishi T. 3D-culture system for heart regeneration and cardiac medicine. Biomed Res Int. 2013;2013:895967.

15. Brandao KO, Tabel VA, Atsma DE, Mummery CL, Davis RP. Human pluripotent stem cell models of cardiac disease: from mechanisms to therapies. Dis Model Mech. 2017;10(9):1039-59.

16. Cashman TJ, Josowitz R, Johnson BV, Gelb BD, Costa KD. Human engineered cardiac tissues created using induced pluripotent stem cells reveal functional characteristics of BRAF-mediated hypertrophic cardiomyopathy. PLoS One. 2016;11(1):e0146697.

17. Wang J, Chen A, Lieu DK, Karakikes I, Chen G, Keung W, et al. Effect of engineered anisotropy on the susceptibility of human pluripotent stem cell-derived ventricular cardiomyocytes to arrhythmias. Biomaterials. 2013;34(35):8878-86.

18. Shum AM, Che H, Wong AO, Zhang C, Wu H, Chan CW, et al. A micropatterned human pluripotent stem cell-based ventricular cardiac anisotropic sheet for visualizing drug-induced arrhythmogenicity. Adv Mater. 2017;29(1):1602448.

19. Chen A, Lieu DK, Freschauf L, Lew V, Sharma H, Wang J, et al. Shrink-film configurable multiscale wrinkles for functional alignment of human embryonic stem cells and their cardiac derivatives. Adv Mater. 2011;23(48):5785-91.

20. Turnbull IC, Karakikes I, Serrao GW, Backeris P, Lee JJ, Xie C, et al. Advancing functional engineered cardiac tissues toward a preclinical model of human myocardium. FASEB J. 2014;28(2):644-54.

21. Cashman TJ, Josowitz R, Gelb BD, Li RA, Dubois NC, Costa KD. Construction of defined human engineered cardiac tissues to study mechanisms of cardiac cell therapy. J Vis Exp. 2016;(109):e53447.

22. Weng Z, Kong CW, Ren L, Karakikes I, Geng L, He J, et al. A simple, costeffective but highly efficient system for deriving ventricular cardiomyocytes from human pluripotent stem cells. Stem Cells Dev. 2014;23(14):1704-16.

23. Puccio H, Simon D, Cossee M, Criqui-Filipe P, Tiziano F, Melki J, et al. Mouse models for Friedreich ataxia exhibit cardiomyopathy, sensory nerve defect and Fe-S enzyme deficiency followed by intramitochondrial iron deposits. Nat Genet. 2001:27(2):181-6.

24. Al-Mahdawi S, Pinto RM, Varshney D, Lawrence L, Lowrie MB, Hughes S, et al. GAA repeat expansion mutation mouse models of Friedreich ataxia exhibit oxidative stress leading to progressive neuronal and cardiac pathology. Genomics. 2006;88(5):580-90.

25. Miranda CJ, Santos MM, Ohshima K, Smith J, Li L, Bunting M, et al. Frataxin knockin mouse. FEBS Lett. 2002;512(1-3):291-7.

26. Chandran V, Gao K, Swarup V, Versano R, Dong H, Jordan MC, et al. Inducible and reversible phenotypes in a novel mouse model of Friedreich's Ataxia. Elife. 2017;6:e30054.

27. Goffart S, von Kleist-Retzow JC, Wiesner RJ. Regulation of mitochondrial proliferation in the heart: power-plant failure contributes to cardiac failure in hypertrophy. Cardiovasc Res. 2004;64(2):198-207.

\section{Publisher's Note}

Springer Nature remains neutral with regard to jurisdictional claims in published maps and institutional affiliations.

\section{Ready to submit your research? Choose BMC and benefit from:}

- fast, convenient online submission

- thorough peer review by experienced researchers in your field

- rapid publication on acceptance

- support for research data, including large and complex data types

- gold Open Access which fosters wider collaboration and increased citations

- maximum visibility for your research: over $100 \mathrm{M}$ website views per year

At BMC, research is always in progress.

Learn more biomedcentral.com/submissions 